\title{
Calculation of Start-Oscillation-Current for Lossy Gyrotron Traveling-Wave Tube (Gyro-TWT) Using Linear Traveling-Wave Tube (TWT) Parameter Conversions
}

\author{
Heather H. Song \\ Department of Electrical and Computer Engineering, University of Colorado, Colorado Springs, USA. \\ Email: hsong@uccs.edu
}

Received November $13^{\text {th }}, 2012$; revised December $13^{\text {th }}$, 2012; accepted December $25^{\text {th }}, 2012$

\begin{abstract}
The start-oscillation-current of a gyro-TWT (gyrotron traveling-wave tube) determines the stable operating current level of the device. The amplifier is susceptible to oscillations when the operating current level is higher than the start-oscillation current. There are several ways of calculating the start-oscillation current, including using the linear and nonlinear theory of a gyro-TWT. In this paper, a simple way of determining the start-oscillation current of lossy gyro-TWT is introduced. The linear TWT parameters that include the effects of synchronism, loss, and gain, were converted to gyro-TWT parameters to calculate the start-oscillation-current. The dependence on magnetic field, loss, and beam alpha was investigated. Calculations were carried out for a V-band gyro-TWT for both operating and competing modes. The proposed method of calculating the start-oscillation current provides a simple and fast way to estimate the oscillation conditions and can be used for the design process of a gyro-TWT.
\end{abstract}

Keywords: Start-Oscillation-Current; Gyro-TWT; TWT; Lossy; Stable; Competing Mode

\section{Introduction}

The gyro-TWT (gyrotron traveling-wave tube) has long been viewed as an extremely promising device due to its high-power and broadband capabilities. Potential applications include radar, communication, surveillance, and scientific research [1]. However, in order for gyro-TWT to work properly, the interaction with competing mode must be suppressed. The beam current level where the unwanted oscillation takes place is called the "start-oscillation-current" $\left(I_{s}\right)^{\prime}$ for gyro-TWT. Therefore in gyroTWT, it is critical to operate the amplifier below $I_{s}$ to ensure stability of the device. One way to increase $I_{s}$ is to apply loss to the gyro-TWT circuit. Calculation results of $I_{S}$ employing linear theory [2-4] and nonlinear theory [5] were reported for lossy gyro-TWT. However, using these methods require in-depth analysis on linear and nonlinear theories of gyro-TWT. In this paper, a simple method of obtaining $I_{S}$ for lossy gyro-TWT by using the linear-TWT parameters is introduced. By using the linear TWT parameter conversions, the expression for $I_{s}$ for the gyroTWT was obtained. The parameter conversion process and the calculation results for $I_{s}$ for gyro-TWT are presented.

\section{Conversion of Linear TWT Parameters to Gyro-TWT}

The gain parameter of a linear lossy TWT that corresponds to the start-oscillation condition can be expressed by Equation (1) [6]. For gyro-TWT, the gain parameter is described as Equation (2) [7]. By combining Equation (1) used in a linear TWT and Equation (2) used in a gyro-TWT (by setting $C_{s t}=C_{g}$ ), the start oscillation current for lossy gyro-TWT can be expressed by Equation (3).

$$
\begin{gathered}
C_{s t}=0.0112 \frac{L_{d B}}{N}\left(1+\frac{1013}{L_{d B}^{2}}\right) \\
C_{g}=\left(\frac{k_{c}^{4}}{2 k_{b}^{4}} I_{b} F_{m n} \varepsilon_{v}\right)^{\frac{1}{3}} \\
I_{s}=\frac{2 k_{b}^{4}}{F_{m n} \varepsilon_{v} k_{c}^{4}}\left(0.0112 \frac{L_{d B}}{N}\right)^{3}\left(1+\frac{1013}{L_{d B}^{2}}\right)^{3}
\end{gathered}
$$

Here $L_{d B}$ is the total loss of the circuit in $d B, N$ is the circuit length in wavelength, $k_{c}$ is the cutoff wavenumber $\left(\omega_{c} / c\right), k_{b}$ is the beam wavenumber $\left((\omega-\Omega) / v_{z}\right), I_{b}$ is 
the beam current, $F_{m n}$ is defined in Equation (4), and $\varepsilon_{v}$ is defined in Equation (5).

$$
\begin{gathered}
F_{m n}=\frac{J_{1-m}^{2}\left(k_{m n} R_{g} / a\right)}{k_{m n}^{2} J_{m}^{2}\left(k_{m n}\right)\left(1-m^{2} / k_{m n}^{2}\right)} \\
\varepsilon_{v}=\frac{2.348 \times 10^{-4}}{\gamma \beta_{z}}\left(\frac{\beta_{\perp}}{\beta_{z}}\right)^{2}\left(J_{1}^{\prime}\left(\frac{\beta_{\perp} \omega_{c}}{\Omega_{c}}\right)\right)^{2}
\end{gathered}
$$

Here $J_{m}$ is the Bessel function of order $m, k_{m n}$ is the $m^{\text {th }}$ Bessel root defined by $J_{m}^{\prime}\left(k_{m n}\right)=0, n$ is the radial mode number, $m$ is the azimuthal mode number, $R_{a}$ is the guiding center radius, $a$ is the waveguide radius, $\beta_{z}$ is the axial velocity normalized by the speed of light, $\beta_{\perp}$ is the transverse velocity normalized by the speed of light, $\omega_{c}$ is the cutoff frequency of the waveguide, and $\Omega_{c}$ is the relativistic cyclotron frequency. The comparison between critical parameters of linear and gyro-TWT is shown in Table 1.

\section{Calculated Results and Discussion}

In order to validate the $I_{s}$ calculation method proposed above, a V-band (60 GHz) $\mathrm{TE}_{11}$ gyro-TWT was chosen to evaluate the $I_{s}$ values. Figure 1 shows the dispersion diagram of the V-band TE 11 gyro-TWT for $\alpha=0.85, V_{b}=$ $100 \mathrm{kV}$, and $B_{o} / B_{g}=1.0$. The waveguide mode expressed as $\omega^{2}=\omega_{c}^{2}+k_{z}^{2} c^{2}$ is shown in parabolas and the beam mode which can be described as $\omega=s \Omega_{c}+k_{z} v_{z}$ is shown in straight lines up to fourth harmonic. Here, $\omega$ is the frequency, $k_{z}$ is the axial wavenumber, $c$ is the speed of light, $s$ is the harmonic number, and $v_{z}$ is the axial velocity of the beam. The operating point is where the $\mathrm{TE}_{11}$ waveguide mode grazes with the $s=1$ beam mode. The possible competing mode interactions occur when the waveguide mode intersects with the beam mode. These include $\mathrm{TE}_{11}$ and $\mathrm{TE}_{21}$ with $s=2, \mathrm{TE}_{01}$ with $s=3$, and $\mathrm{TE}_{02}$ with $s=4$ beam modes. The specification

Table 1. Conversion of linear TWT parameters to gyroTWT [7-8].

\begin{tabular}{ccc}
\hline Parameter & Linear TWT & Gyro-TWT \\
\hline Gain & $C=\left(\frac{Z_{0} I_{0}}{4 V_{b}}\right)^{\frac{1}{3}}$ & $C_{g}=\left(\frac{k_{c}^{4}}{2 k_{b}^{4}} I_{b} F_{m n} \varepsilon_{v}\right)^{\frac{1}{3}}$ \\
Synchronism & $b=\frac{1}{C}\left(\frac{u_{0}}{v_{p}}-1\right)$ & $g=\frac{k_{g}-k_{b}}{k_{b} C_{g}}$ \\
Loss & $d=0.0184 \frac{L}{C}$ & $d=0.0184 \frac{L}{C_{g}}$
\end{tabular}

$Z_{0}$ : Circuit impedance; $v_{p}$ : Phase velocity; $V_{b}$ : Beam voltage; $L$ : Loss per wavelength; $u_{0}$ : Beam velocity; $k_{a}$ : Waveguide wavenumber.

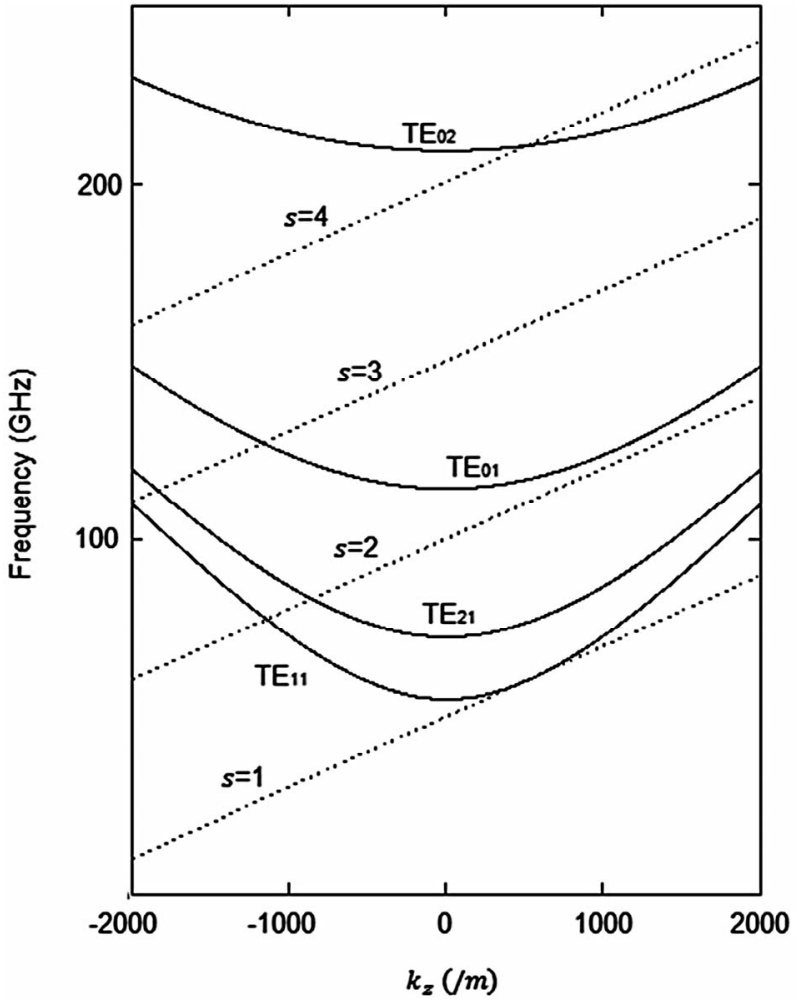

Figure 1. Dispersion diagram of a $\mathrm{TE}_{11} 60 \mathrm{GHz}$ gyro-TWT for $\alpha=0.85, V_{b}=100 \mathrm{kV}$, and $B_{o} / B_{g}=1.0$. Waveguide modes $\left(\mathrm{TE}_{11}, \mathrm{TE}_{21}, \mathrm{TE}_{01}, \mathrm{TE}_{02}\right)$ and beam modes $(s=1,2,3$, 4) are shown.

of the V-band $\mathrm{TE}_{11}$ gyro-TWT is described in Table 2. The calculated $I_{s}$ using Equation (3) is shown in Figures 2-5 under various conditions. Figure 2 shows dependence of $I_{s}$ on circuit loss, $L_{d B}$, for several values of beam velocity ratio, $\alpha$, for the operating $\mathrm{TE}_{11}$ mode with $B_{o} / B_{g}=1.5$ and $V_{b}=100 \mathrm{kV}$. The $B_{o} / B_{g}$ indicates operating magnetic field, $B_{o}$, normalized by the grazing magnetic field, $B_{g}$. As $L_{d B}$ increases, $I_{s}$ increases which indicates that with higher value of $L_{d B}$, the device becomes more stable. For fixed value of $L_{d B}, I_{s}$ increases as $\alpha$ decreases. This indicates that the loss stabilizes the device and the gyro-TWT becomes unstable for higher values of $\alpha$. Figure 3 describes $I_{s}$ change with $B_{o} / B_{g}$ of the operating $\mathrm{TE}_{11}$ mode for several values of beam voltage, $V_{b}$, for fixed values of $\alpha=0.85$ and $L_{d B}=100 \mathrm{~dB}$. For $B_{o} / B_{g}<1.1, I_{s}$ decreases as $B_{o} / B_{g}$ increases. For fixed value of $B_{o} / B_{g}, I_{s}$ is higher for higher $V_{b}$ when $B_{o} / B_{g}<1.1$. For $B_{o} / B_{g}>1.1$, higher beam voltage makes the device unstable and as the operating magnetic field increases the device becomes more stable due to increasing $\left|k_{z}\right|$. Figure 4 shows $I_{s}$ as a function $B_{o} / B_{g}$ for several values of $\alpha$ and fixed values of $V_{b}=100 \mathrm{kV}$ and $L_{d B}=100 \mathrm{~dB}$. For $B_{o} / B_{g}<1.1, I_{s}$ decreases as $B_{o} / B_{g}$ increases. For fixed value of $B_{o} / B_{g}, I_{s}$ in 
Table 2. Specifications of the V-band $\mathrm{TE}_{11}$ gyro-TWT.

\begin{tabular}{|c|c|}
\hline Parameter & Value \\
\hline Beam voltage, $V_{b}$ & $100 \mathrm{kV}$ \\
\hline Beam current, $I_{b}$ & $3 \mathrm{~A}$ \\
\hline Velocity pitch, $\alpha$ & 0.85 \\
\hline Operating mode & $\mathrm{TE}_{11}$ \\
\hline Cyclotron harmonic & $s=1$ \\
\hline Magnetic field, $B_{o}$ & $21.4 \mathrm{kG}$ \\
\hline$B_{o} / B_{g}$ & 0.995 \\
\hline Circuit radius, $r_{w}$ & $0.16 \mathrm{~cm}$ \\
\hline$r_{c} / r_{w}$ & 0.361 \\
\hline Circuit length & $14 \mathrm{~cm}$ \\
\hline Lamor radius, $r_{L}$ & $0.034 \mathrm{~cm}$ \\
\hline Cutoff frequency & $55 \mathrm{GHz}$ \\
\hline Efficiency & $24.5 \%$ \\
\hline Output power & $78.2 \mathrm{~W}$ \\
\hline Gain & $41 \mathrm{~dB}$ \\
\hline Bandwidth & $5 \%$ \\
\hline
\end{tabular}

$r_{c}$ : Guiding center radius.

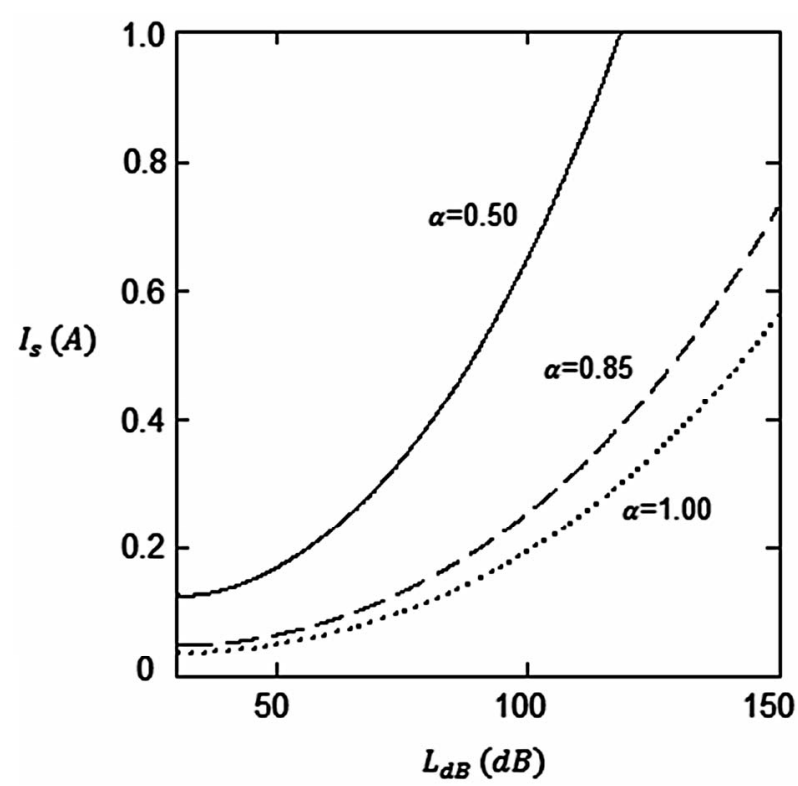

Figure 2. Start-oscillation-current, $I_{s}$, for the operating $\mathrm{TE}_{11}$ mode as a function of loss, $L_{d B}$, for different values of beam velocity ratio, $\alpha$, and the fixed value of the operating magnetic field normalized by the grazing magnetic field, $\boldsymbol{B}_{d} / \boldsymbol{B}_{g}=$ 1.5 , and beam voltage, $V_{b}=100 \mathrm{kV}$.

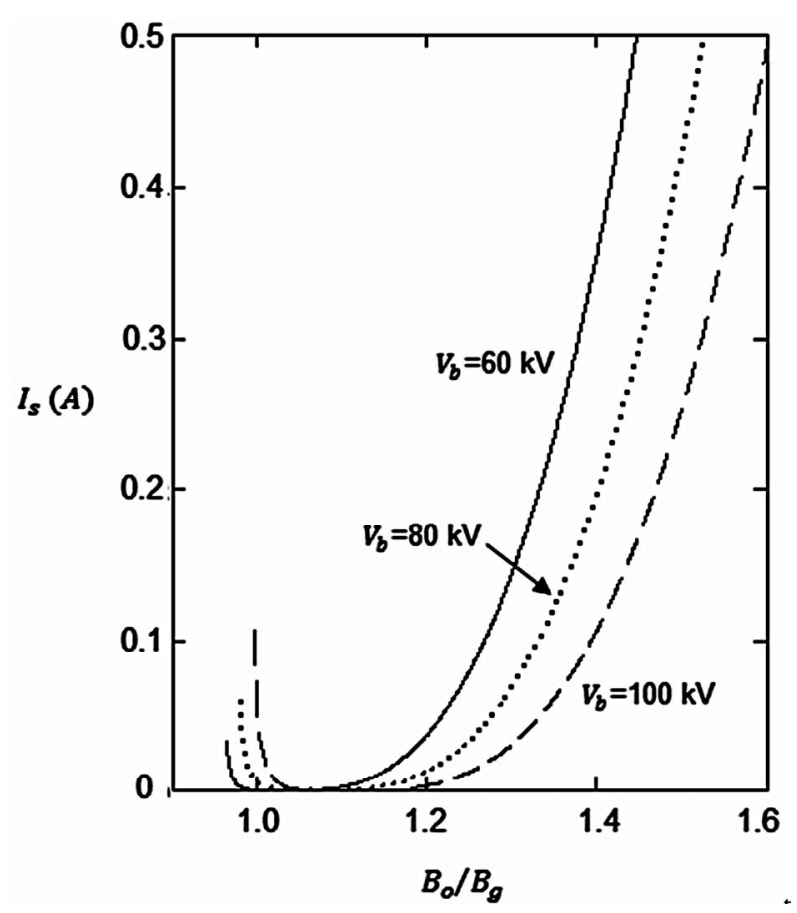

Figure 3. Start-oscillation-current, $I_{s}$, for the operating $\mathrm{TE}_{11}$ mode as a function of $B_{d} / B_{g}$ for different values of beam voltage, $V_{b}$, and fixed values of $\alpha=0.85$ and $L_{d B}=100 \mathrm{~dB}$.

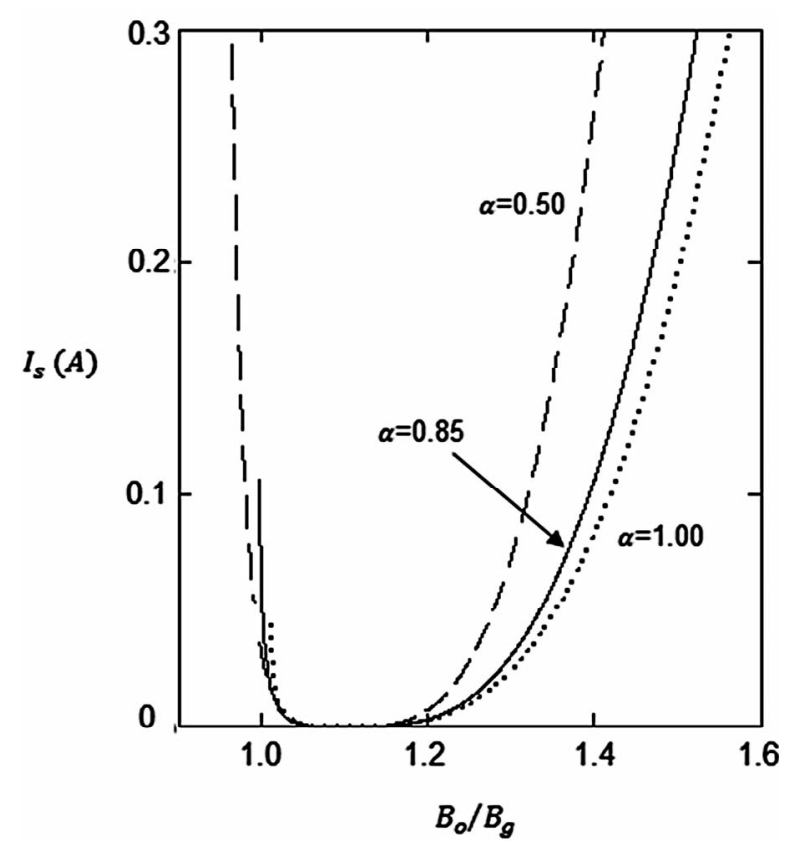

Figure 4. Start-oscillation-current, $I_{s}$, for the operating $\mathrm{TE}_{11}$ mode as a function of $B_{o} / B_{g}$ for different values of $\alpha$ and fixed values of $V_{b}=100 \mathrm{kV}$ and $L_{d B}=100 \mathrm{~dB}$.

creases as $\alpha$ increases for $B_{o} / B_{g}<1.1$. For $B_{o} / B_{g}>1.1$, device is more stable for lower alpha because when the perpendicular component of the velocity decreases, the gyro-TWT beam-wave interaction becomes 


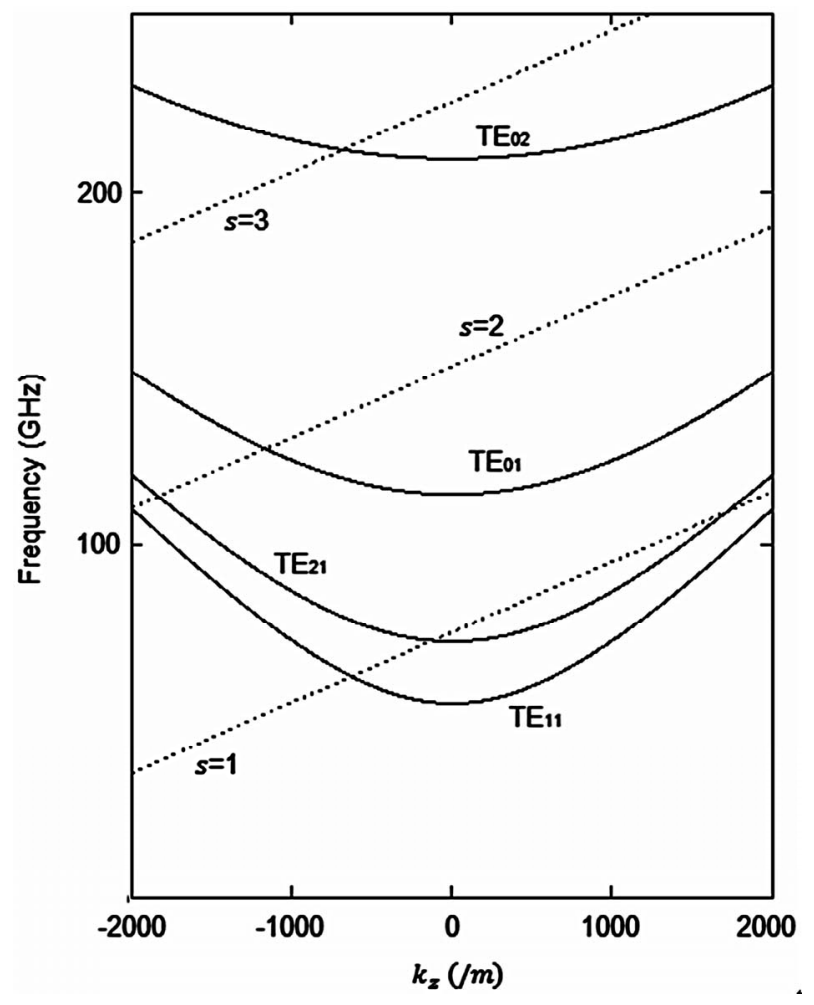

Figure 5. Dispersion diagram of a TE $1160 \mathrm{GHz}$ gyro-TWT for $\alpha=0.85, V_{b}=100 \mathrm{kV}$, and $B_{o} / B_{g}=1.5$. Waveguide modes $\left(\mathrm{TE}_{11}, \mathrm{TE}_{21}, \mathrm{TE}_{01}, \mathrm{TE}_{02}\right)$ and beam modes $(\mathrm{s}=1,2,3)$ are shown.

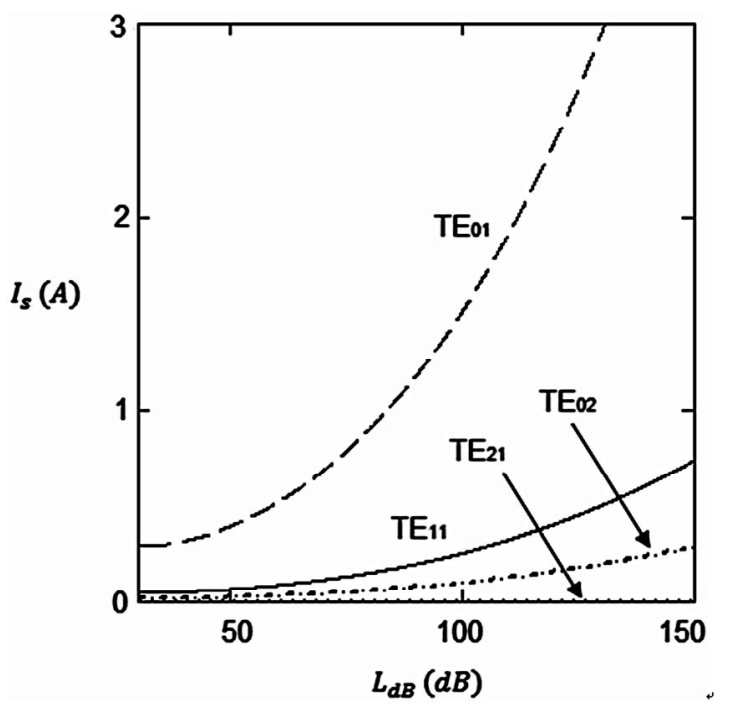

Figure 6. Start-oscillation-current, $I_{s}$, as a function of loss, $L_{d B}$, for different modes. Fixed values of $\alpha=0.85, V_{b}=100$ $\mathrm{kV}$, and $B_{o} / B_{g}=1.5$ were assumed.

weaker. Figure 5 shows the dispersion diagram of the device for $\alpha=0.85, V_{b}=100 \mathrm{kV}$, and $B_{o} / B_{g}=1.5$. Figure 6 describes $I_{s}$ as a function of $L_{d B}$ for four different modes: $\mathrm{TE}_{11}, \mathrm{TE}_{21}, \mathrm{TE}_{01}$, and $\mathrm{TE}_{02}$. Fixed values of $\alpha$
$=0.85, V_{b}=100 \mathrm{kV}$, and $B_{o} / B_{g}=1.5$ were assumed. The lowest $I_{s}$ occurs for the $\mathrm{TE}_{21}$ and the $\mathrm{TE}_{01}$ mode exhibits the highest $I_{s}$ value. As can be seen in Figure 5, this is due to $T E_{21}$ mode having the lowest $\left|k_{z}\right|$ value and the $\mathrm{TE}_{01}$ mode having the highest $\left|k_{z}\right|$ value at the intersection of the beam-wave dispersion diagram. The $I_{s}$ of the $\mathrm{TE}_{01}$ mode is the most sensitive to $L_{d B}$ variation.

\section{Summary and Conclusion}

In this paper, an expression for $I_{s}$ for lossy gyro-TWT was derived using linear TWT parameter conversions. For V-band $\mathrm{TE}_{11}$ gyro-TWT, $I_{s}$ was calculated for various parameters including loss, beam voltage, magnetic field, and beam velocity ratio. The method introduced in this paper can be used to quickly estimate the $I_{s}$ values of a lossy gyro-TWT.

\section{REFERENCES}

[1] H. H. Song, D. B. McDermott, Y. Hirata, L. R. Barnett, C. W. Domier, H. L. Hsu, T. H. Chang, W. C. Tsai, K. R. Chu and N. C. Luhmann Jr., "Theory and Experiment of a 94 GHz Gyrotron Traveling-Wave Amplifier," Physics of Plasmas, Vol. 11, No. 5, 2004, pp. 2935-2941. doi:10.1063/1.1690764

[2] Q. S. Wang, D. B. McDermott and N. C. Luhmann Jr., "Demonstration of Marginal Stability Theory by a 200kW Second-Harmonic Gyro-TWT Amplifier,” Physical Review Letters, Vol. 75, No. 23, 1995, pp. 4322-4355. doi:10.1103/PhysRevLett.75.4322

[3] C. S. Kou, Q. S. Wang, D. B. McDermott, A. T. Lin, K. R. Chu and N. C. Luhmann Jr., "High-Power Harmonic GyroTWT's-Part I: Linear Theory and Oscillation Study," IEEE Transactions on Plasma Science, Vol. 20, No. 3, 1992, pp. 155-162. doi:10.1109/27.142815

[4] Y. Y. Lau, K. R. Chu, L. R. Barnett and V. L. Granatstein, "Gyrotron Traveling Wave Amplifier: I. Analysis of Oscillations," International Journal of Infrared and Millimeter Waves, Vol. 2, No. 3, 1981, pp. 373-393. doi:10.1007/BF01007408

[5] W. C. Tsai, T. H. Chang, N. C. Chen, K. R. Chu, H. H. Song and N. C. Luhmann Jr., "Absolute Instabilities in a High-Order-Mode Gyrotron Traveling-Wave-Amplifier," Physical Review E, Vol. 70, No. 5, 2004, Article ID: 056402. doi:10.1103/PhysRevE.70.056402

[6] R. W. Grow and D. R. Gunderson, "Starting Conditions for Backward-Wave Oscillators with Large Loss and Large Space Charge," IEEE Transactions on Electron Devices, Vol. 17, No. 12, 1970, pp. 1032-1039. doi:10.1109/T-ED.1970.17123

[7] M. Caplan, “The Gyrotron: An Application of the Relativistic Bunching of Electrons to the Generation of Intense Millimeter Microwave Radiation,” Ph.D. Thesis, University of California, Los Angeles, 1986.

[8] A. S. Gilmour, "Principles of Traveling Wave Tubes," Artech House Inc., Norwood, 1994, pp. 273-305. 